\section{Gas phase homolytic bond dissociation enthalpies of common laboratory solvents: A G4 theoretical study}

\section{Sierra Rayne ${ }^{a,{ }^{*}}$ and Kaya Forest ${ }^{\mathrm{b}}$}

Ground and excited state hydrogen atom abstraction reactions play a fundamental role in chemistry. In particular, hydrogen atom abstraction from various laboratory solvents is exploited as a means for synthesizing new materials, as well as conducting mechanistic studies.[1,2] In general, the rate of hydrogen atom abstraction by neutral or radical species in solution correlates with the bond dissociation enthalpy (BDE) of the solvent. This relationship can be described by the Bell-Evans-Polanyi [3] relationship coupled with the Hammond Postulate,[4] which can be expressed as $E_{a}=\alpha(B D E)+\beta$, where $E_{a}$ is the activation energy for the single step hydrogen atom abstraction reaction, and $\alpha$ and $\beta$ are constants specific for a particular type of bond being broken in the rate determining step (e.g., C-H) and other experimental conditions. The rate of reaction, $\mathrm{k}_{\mathrm{r}}$, can be related to the activation energy via the Arrhenius equation,

$\mathrm{k}_{\mathrm{r}}=A e^{-\mathrm{Ea} / \mathrm{RT}}$, where $\mathrm{R}$ is the gas constant, $\mathrm{T}$ is the absolute temperature, and $A$ is the pre-exponential factor.

Consequently, standard sources [5] offer BDEs of various organic and inorganic compounds for general use in reaction modeling. Given the wide range of possible substrates for hydrogen abstraction reactions and costs of experimental work, high level computational methods are increasingly employed for BDE estimates.[6] Often, with the near chemical accuracy achieved by modern composite computational methods, the theoretical results can also be used to assess potential errors in the experimental data. In the current work, we conducted gas-phase standard state (298.15 $\mathrm{K} ; 1 \mathrm{~atm}$ ) Gaussian-4 (G4) [7] level calculations using Gaussian 09 [8] on a suite of common laboratory solvents (Figure 1), and compare the G4 BDE estimates to the recommended experimental values. Calculations used gas phase starting geometries obtained with the PM6 semiempirical method [9] as implemented in MOPAC 2009 (http://www.openmopac.net/; v. 9.281). All molecular enthalpies and free energies include zero point and thermal corrections. Only the lowest energy conformation of each molecule was considered. All optimized structures were confirmed as true minima by vibrational analysis at the same level.

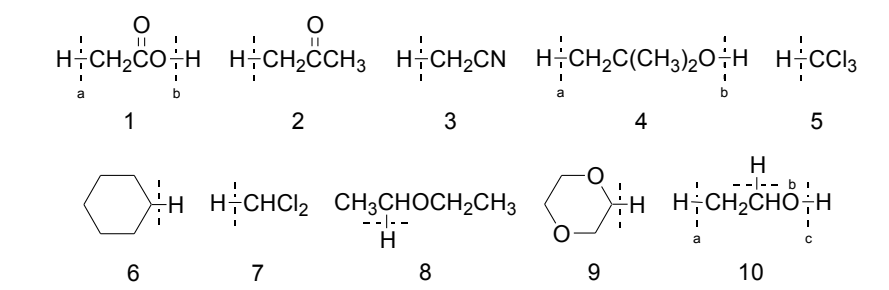

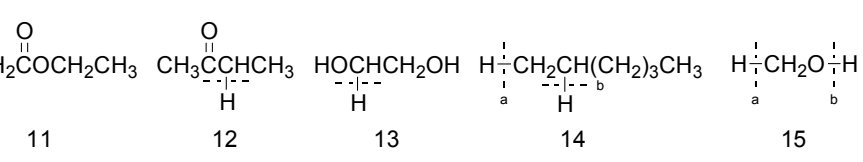

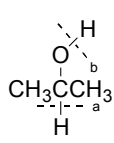

18

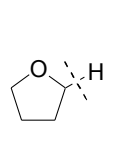

19

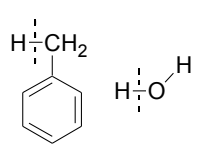

$20 \quad 21$
Fig. 1. Structures and homolytic bond cleavage paths for the compounds under consideration.

We find good agreement between the recommended experimental values [5] and our G4 estimates, having a mean signed error of $0.2 \mathrm{kcal} / \mathrm{mol}$, a mean absolute error of $1.2 \mathrm{kcal} / \mathrm{mol}$, and a root mean squared error of 1.5 $\mathrm{kcal} / \mathrm{mol}$ (Table 1). In a number of cases, the recommended experimental $\mathrm{BDE}$ represents a preferred single experimental value, where several possible experimental values exist. In such situations, the G4 results presented herein may assist in re-evaluating which, if any, of the potential experimental values put forward for each BDE under consideration are likely to be most accurate. For example, a difference of $1.9 \mathrm{kcal} / \mathrm{mol}$ exists between the recommended $\mathrm{C}-\mathrm{H}$ BDE for cyclohexane $(99.5 \mathrm{kcal} / \mathrm{mol})$ and our G4 value $(97.6 \mathrm{kcal} / \mathrm{mol})$. However, the recommended cyclohexane C$\mathrm{H} \mathrm{BDE}$ is the highest experimental value among five reports in the compendium (96.2, 96.4 $0.6,97.6,98$, and $99.5 \mathrm{kcal} / \mathrm{mol}) .[5]$ If one assumes that the $\mathrm{G} 4$ level calculations should achieve near chemical accuracy $(<1 \mathrm{kcal} / \mathrm{mol}$ error) for BDE estimates, then the experimental datapoints of 97.6 and $98.0 \mathrm{kcal} / \mathrm{mol}$ may better represent the preferred $\mathrm{BDE}$ for cyclohexane. In this context, we intend the current computational note as a potentially useful resource in any future reevaluations of the

\begin{tabular}{|c|c|c|c|c|}
\hline ID & compound & bond & expt. $D^{\circ}{ }_{298 K,(g)}{ }^{a}$ & $\mathrm{G} \mathrm{D}_{298 \mathrm{~K},(\mathrm{~g})}$ \\
\hline \multirow[t]{2}{*}{1} & acetic acid & a & $95.3 \pm 2.9$ & 97.9 \\
\hline & & b & 106.4 & 109.3 \\
\hline 2 & acetone & & $95.9 \pm 0.7$ & 95.4 \\
\hline 3 & acetonitrile & & 96.0 & 96.3 \\
\hline \multirow[t]{2}{*}{4} & t-butanol & a & $100.0 \pm 2.0$ & 101.2 \\
\hline & & b & $106.3 \pm 0.7$ & 105.4 \\
\hline 5 & chloroform & & $93.8 \pm 0.6$ & 92.4 \\
\hline 6 & cyclohexane & & 99.5 & 97.6 \\
\hline 7 & dichloromethane & & $97.3 \pm 1.0$ & 95.3 \\
\hline 8 & diethyl ether & & 93.0 & 93.8 \\
\hline 9 & dioxane & & 96.6 & 95.7 \\
\hline \multirow[t]{3}{*}{10} & ethanol & a & 101.3 & 101.9 \\
\hline & & b & 94.8 & 94.3 \\
\hline & & c & $104.6 \pm 0.8$ & 103.5 \\
\hline 11 & ethyl acetate & & 96.0 & 97.0 \\
\hline 12 & ethyl methyl ketone & & $92.3 \pm 1.7$ & 90.2 \\
\hline 13 & ethylene glycol & & 92.1 & 95.4 \\
\hline \multirow[t]{2}{*}{14} & hexane & a & 99.0 & 99.6 \\
\hline & & b & 98.0 & 97.1 \\
\hline \multirow[t]{2}{*}{15} & methanol & a & $96.1 \pm 0.2$ & 96.0 \\
\hline & & b & $104.2 \pm 0.9$ & 104.4 \\
\hline 16 & nitromethane & & 97.4 & 100.6 \\
\hline \multirow[t]{2}{*}{17} & pentane & a & 100.2 & 100.0 \\
\hline & & b & 99.2 & 97.5 \\
\hline \multirow[t]{2}{*}{18} & 2-propanol & a & $91.0 \pm 1.0$ & 93.3 \\
\hline & & b & $105.7 \pm 0.7$ & 105.3 \\
\hline 19 & tetrahydrofuran & & $92.1 \pm 1.6$ & 92.2 \\
\hline 20 & toluene & & $88.5 \pm 1.5$ & 89.8 \\
\hline 21 & water & & $118.8 \pm 0.1$ & 118.1 \\
\hline
\end{tabular}
preferred BDEs for these common laboratory solvents.

Table 1. Experimental and G4 estimated bond dissociation enthalpies for various common laboratory solvents. Values are in $\mathrm{kcal} / \mathrm{mol}$.

a from ref. [5].

\section{Acknowledgements}

This work was made possible by the facilities of the Western Canada Research Grid (WestGrid:www.westgrid.ca; project 100185), the Shared Hierarchical Academic Research Computing Network (SHARCNET: www.sharcnet.ca; project sn4612), and Compute/Calcul Canada.

\section{References and Notes}

a Ecologica Research, 412-3311 Wilson Street, Penticton, British Columbia, V2A 8J3, Canada

${ }^{\mathrm{b}}$ Department of Chemistry, Okanagan College, 583 Duncan Avenue West, Penticton, British Columbia, V2A 8E1, Canada

* Corresponding author: rayne.sierra@gmail.com

[1] Carey, F.A. "Advanced Organic Chemistry, Part A: Structure and Mechanisms.", Springer: New York, NY, USA, 2000.

[2] Carey, F.A. "Advanced Organic Chemistry, Part B: Reactions and Synthesis.", Springer: New York, NY, USA, 2007.

[3] Evans, M.G.; Polanyi, M. "Inertia and driving force of chemical reactions.", Trans. Faraday Soc. 1938, 34, 11-23.

[4] Hammond, G.S. "A correlation of reaction rates.", J. Am. Chem. Soc. 1955, 77, 334-338.

[5] Luo, Y.R. "Handbook of Bond Dissociation Energies in Organic Compounds.”, CRC Press: Boca Raton, FL, USA, 2002.

[6] Zhang, I.Y.; $\mathrm{Wu}, \mathrm{J}$.; $\mathrm{Xu}, \mathrm{X}$. "Trends in $\mathrm{R}-\mathrm{X}$ bond dissociation energies $(\mathrm{R} \bullet=\mathrm{Me}, \mathrm{Et}$, i-Pr, t-Bu, $\mathrm{X} \cdot=\mathrm{H}, \mathrm{Me}, \mathrm{Cl}, \mathrm{OH})$. ., J. Chem. Theory Comput., doi: $10.1021 /$ ct $100010 d$.

[7] Curtiss, L.A.; Redfern, P.C.; Raghavachari, K. "Gaussian-4 theory." J. 
Chem. Phys. 2007, 126, 084108.

[8] Gaussian 09, Revision A.1, Frisch, M.J.; Trucks, G.W.; Schlegel, H.B.; Scuseria, G.E.; Robb, M.A.; Cheeseman, J.R.; Scalmani, G.; Barone, V.; Mennucci, B.; Petersson, G.A.; Nakatsuji, H.; Caricato, M.; Li, X.;

Hratchian, H. P.; Izmaylov, A. F.; Bloino, J.; Zheng, G.; Sonnenberg, J.L.;

Hada, M.; Ehara, M.; Toyota, K.; Fukuda, R.; Hasegawa, J.; Ishida, M.;

Nakajima, T.; Honda, Y.; Kitao, O.; Nakai, H.; Vreven, T.; Montgomery, Jr., J.A.; Peralta, J.E.; Ogliaro, F.; Bearpark, M.; Heyd, J.J.; Brothers, E.;

Kudin, K.N.; Staroverov, V.N.; Kobayashi, R.; Normand, J.; Raghavachari,

K.; Rendell, A.; Burant, J.C.; Iyengar, S.S.; Tomasi, J.; Cossi, M.; Rega, N.;

Millam, N.J.; Klene, M.; Knox, J.E.; Cross, J.B.; Bakken, V.; Adamo, C.;

Jaramillo, J.; Gomperts, R.; Stratmann, R.E.; Yazyev, O.; Austin, A.J.;

Cammi, R.; Pomelli, C.; Ochterski, J.W.; Martin, R.L.; Morokuma, K.;

Zakrzewski, V.G.; Voth, G.A.; Salvador, P.; Dannenberg, J.J.; Dapprich, S.; Daniels, A. D.; Farkas, O.; Foresman, J.B.; Ortiz, J.V.; Cioslowski, J.; Fox, D.J. Gaussian, Inc., Wallingford, CT, USA, 2009.

[9] Stewart, J.J.P. "Optimization of parameters for semiempirical methods. V. Modification of NDDO approximations and application to 70 elements."

J. Mol. Model. 2007, 13, 1173-1213. 